KYUNGPOOK Math. J. 55(2015), 279-287

http://dx.doi.org/10.5666/KMJ.2015.55.2.279

pISSN 1225-6951 eISSN 0454-8124

(C) Kyungpook Mathematical Journal

\title{
Double Domination in the Cartesian and Tensor Products of Graphs
}

\author{
Arnel Marino Cuivillas* \\ Department of Mathematics, Jose Rizal Memorial State University, Dipolog City, \\ 7100, Philippines \\ e-mail : navcui@yahoo.com \\ Sergio R. Canoy, JR. \\ Department of Mathematics and Statistics, Mindanao State University - Iligan In- \\ stitute of Technology, Iligan City, 9200, Philippines \\ e-mail : serge_canoy@yahoo.com
}

Abstract. A subset $S$ of $V(G)$, where $G$ is a graph without isolated vertices, is a double dominating set of $G$ if for each $x \in V(G),\left|N_{G}[x] \cap S\right| \geq 2$. This paper, shows that any positive integers $a, b$ and $n$ with $2 \leq a<b, b \geq 2 a$ and $n \geq b+2 a-2$, can be realized as domination number, double domination number and order, respectively. It also characterize the double dominating sets in the Cartesian and tensor products of two graphs and determine sharp bounds for the double domination numbers of these graphs. In particular, it show that if $G$ and $H$ are any connected non-trivial graphs of orders $n$ and $m$ respectively, then $\gamma_{\times 2}(G \square H) \leq \min \left\{m \gamma_{2}(G), n \gamma_{2}(H)\right\}$, where $\gamma_{2}$, is the 2-domination parameter.

\section{Introduction}

Let $G=(V(G), E(G))$ be a graph. For any vertex $x \in V(G)$, the open neighborhood of $x$ is the set $N_{G}(x)=\{y \in V(G): x y \in E(G)\}$ and the closed neighborhood of $x$ in $G$ is the set $N_{G}[x]=N_{G}(x) \cup\{x\}$. If $X \subseteq V(G)$, the open neighborhood of $X$ in $G$ is the set $N_{G}(X)=\bigcup_{x \in X} N_{G}(x)$. The closed neighborhood of $X$ in $G$ is the set $N_{G}[X]=N_{G}(X) \cup X$.

A subset $S$ of $V(G)$ is a dominating set in $G$ if $N_{G}[S]=S \cup N_{G}(S)=V(G)$

* Corresponding Author.

Received April 20, 2014; accepted September 19, 2014.

2010 Mathematics Subject Classification: 05C69.

Key words and phrases: Domination, double domination, Cartesian, tensor product.

This work is partially funded by the Commission on Higher Education (CHED), Philippines under Faculty Development Program Phase II. 
where $N_{G}(S)=\{v \in V(G): x v \in E(G)$ for some $x \in S\}$. Equivalently, a subset $S$ of $V(G)$ is a dominating set in $G$ if for every $v \in V(G) \backslash S$, there exists $x \in S$ such that $x v \in E(G)$. The minimum cardinality of a dominating set in $G$, denoted by $\gamma(G)$, is the domination number of $G$. Moreover, a subset $S$ of $V(G)$ is a 2dominating set of the graph $G$, if every vertex $v \in V(G) \backslash S$ is adjacent to at least 2 vertices in $S$. The 2-domination number $\gamma_{2}(G)$ is the minimum cardinality of a 2-dominating set of $G$. A 2-dominating set of $G$ with cardinality $\gamma_{2}(G)$ is called a $\gamma_{2}$-set.

Let $G=(V(G), E(G))$ be a graph with no isolated vertices. A subset $S$ of $V(G)$ is a double dominating set of $G$ if for each $x \in V(G),\left|N_{G}[x] \cap S\right| \geq 2$. The double domination number of $G$, denoted by $\gamma_{\times 2}(G)$, is the minimum cardinality of a double dominating set of $G$. A double dominating set of $G$ with cardinality $\gamma_{\times 2}(G)$ is called a $\gamma_{\times 2}$-set.

Double dominating set and double domination number were first defined and introduced by F. Harary and T. W. Haynes in [4] \{as cited in [5]\}. They also established the Nordhaus-Gaddum inequalities for double domination. Blidia, Chellali and Haynes [2] characterized the trees having equal paired and double domination number. Atapour, Khodkar and Sheikholeslami [1] established upper bounds on the double domination subdivision number (the minimum number of edges that must be subdivided in order to increase the $\left.\gamma_{\times 2}(G)\right)$ for arbitrary graphs in terms of vertex degree. Khelifi et al. [6] studied the concept in relation to $\gamma_{\times 2}$-critical graphs (the removal of any edge which increases the $\gamma_{\times 2}(G)$ ). Cuivillas and Canoy [3] characterized and determined sharp bounds for the double dominating sets in the join, corona and lexicographic product of two graphs.

\section{Realization Problem}

It is shown in [3] that $1+\gamma(G) \leq \gamma_{\times 2}(G)$ for any graph $G$ without isolated vertices. Also, by a remark in [3], for any graph $G$ of order $n \geq 2$ without isolated vertices, $2 \leq \gamma_{\times 2}(G) \leq n$. Thus, the following remark is immediate.

Remark 2.1. For any non-trivial graph $G, \gamma(G)<\gamma_{\times 2}(G) \leq|V(G)|$.

Theorem 2.2. Given positive integers $a, b$ and $n$ with $2 \leq a<b, b \geq 2 a$ and $n \geq b+2 a-2$, there exists a connected graph $G$ with $\gamma(G)=a, \gamma_{\times 2}(G)=b$ and $|V(G)|=n$.

Proof. Consider the following cases:

Case 1. $b=2 a$ and $n \geq b+2 a-2$

Suppose $a=2$. Then $b=4$. Let $H$ be a path $\left[x_{1}, y_{1}, v_{1}, x_{2}\right]$ and let $G_{1}$ be a graph obtained from $H$ by adding the vertices $z_{i}$ for $i=1,2$ and the edges $z_{1} y_{1}$, $z_{2} v_{1}$, and $z_{i} x_{i}$ for $i=1,2$. Moreover, let $G_{2}$ be a graph obtained from $G_{1}$ by adding the paths $\left[x_{1}, u_{m}, z_{1}\right]$ for $m=1,2, \ldots, n-b-2 a+2$ (see Figure 1 ). Then the set $\left\{x_{1}, x_{2}\right\}$ is a minimum dominating set of $G_{1}$ and $G_{2}$. Hence $\gamma\left(G_{1}\right)=\gamma\left(G_{2}\right)=2=a$. Also, a minimum double dominating set of $G_{1}$ and $G_{2}$ is the set $\left\{x_{1}, x_{2}\right\} \cup\left\{z_{1}, z_{2}\right\}$. Thus $\gamma_{\times 2}\left(G_{1}\right)=\gamma_{\times 2}\left(G_{2}\right)=2+2=2 a=b$. Now if $n=b+2 a-2$ then $n=6$. Thus 
take $G=G_{1}$, where $\left|V\left(G_{1}\right)\right|=2+2+1+1=n$. If not, take $G=G_{2}$, where $\left|V\left(G_{2}\right)\right|=2+2+1+1+(n-b-2 a+2)=n-b+4=n$.
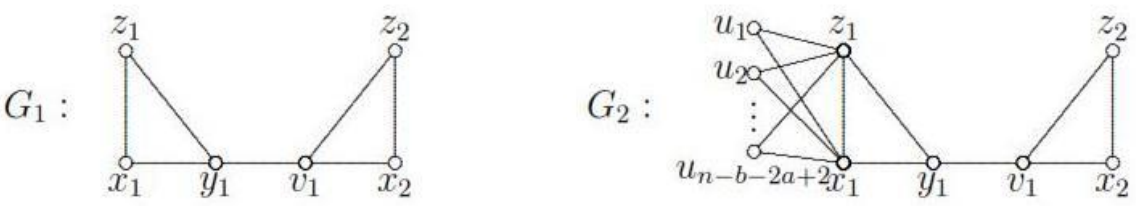

Figure 1: Graphs $G_{1}$ and $G_{2}$

Suppose now that $a>2$. Let $P_{a}$ be the path $\left[x_{1}, x_{2}, \ldots, x_{a-1}, x_{a}\right]$ and let $G_{3}$ be the graph obtained from $P_{a}$ by adding the vertices $z_{i}$ for $i=1,2, \ldots, a$ and replacing the edges $x_{i} x_{i+1}$ by the paths $\left[x_{i}, y_{i}, v_{i+1}, x_{i+1}\right]$ for $i=1,2, \ldots, a-1$, then adding the edges $z_{i} x_{i}$ for $i=1,2, \ldots, a, z_{j} y_{j}$ for $j=1,2, \ldots, a-1$, and $z_{k} v_{k}$ for $k=2,3, \ldots, a$. Moreover, let $G_{4}$ be the graph obtained from $G_{3}$ by adding the paths $\left[x_{1}, u_{m}, z_{1}\right]$ for $m=1,2, \ldots, n-b-2 a+2$ (see Figure 2). Then the set $\left\{x_{1}, x_{2}, \ldots, x_{a-1}, x_{a}\right\}$ is a minimum dominating set of $G_{3}$ and $G_{4}$, while a minimum double dominating set of $G_{3}$ and $G_{4}$ is the set $\left\{x_{1}, x_{2}, \ldots, x_{a-1}, x_{a}\right\} \cup\left\{z_{1}, z_{2}, \ldots, z_{a-1}, z_{a}\right\}$. Hence $\gamma\left(G_{3}\right)=$ $\gamma\left(G_{4}\right)=a$ and $\gamma_{\times 2}\left(G_{3}\right)=\gamma_{\times 2}\left(G_{4}\right)=a+a=2 a=b$. Now if $n=b+2 a-2$, then take $G=G_{3}$, where $\left|V\left(G_{3}\right)\right|=a+a+(a-1)+(a-1)=4 a-2=n$. If $n>b+2 a-2$, then take $G=G_{4}$, where $\left|V\left(G_{4}\right)\right|=a+a+(n-b-2 a+2)+(a-1)+(a-1)=n$.
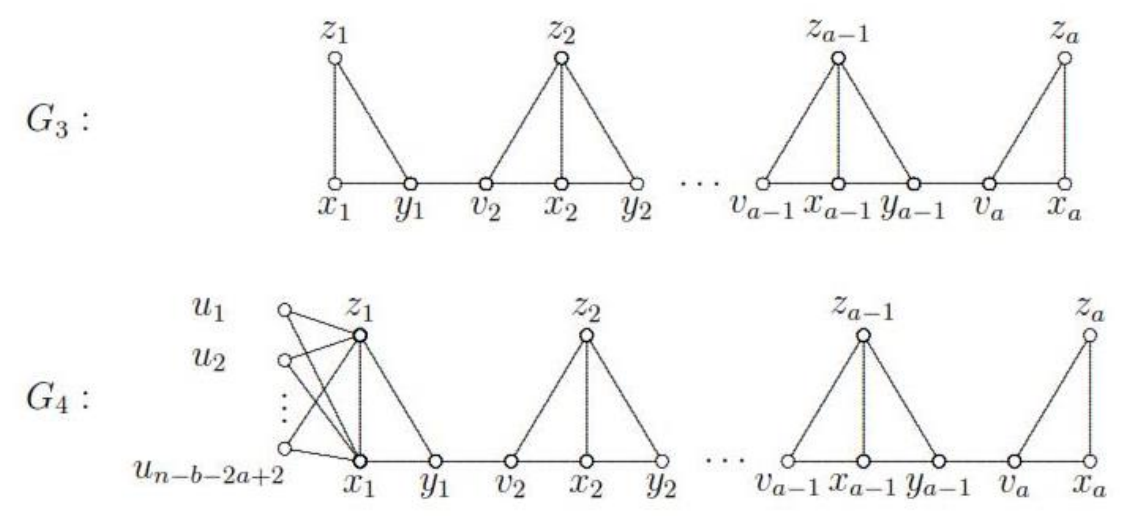

Figure 2: Graphs $G_{3}$ and $G_{4}$

Case 2. $b>2 a$ and $n \geq b+2 a-2$ 
Suppose $a=2$. Consider the graph $G_{5}$ obtained from $G_{1}$ in Figure 1 by adding the vertices $w_{l}$ and the edges $z_{2} w_{l}$ for $l=1,2, \ldots, b-2 a$. Also, let $G_{6}$ be the graph obtained from $G_{5}$ by adding the paths $\left[x_{1}, u_{m}, z_{1}\right]$ for $m=1,2, \ldots, n-b-2 a+2$ (see Figure 3). Then the set $\left\{z_{1}, z_{2}\right\}$ is a $\gamma$-set of $G_{5}$ and $G_{6}$. Hence, $\gamma\left(G_{5}\right)=$ $\gamma\left(G_{6}\right)=2=a$. Also, a minimum double dominating set of $G_{5}$ and $G_{6}$ is the set $\left\{x_{1}, x_{2}\right\} \cup\left\{z_{1}, z_{2}\right\} \cup\left\{w_{1}, w_{2}, \ldots, w_{b-2 a}\right\}$. Thus, $\gamma_{\times 2}\left(G_{5}\right)=\gamma_{\times 2}\left(G_{6}\right)=2+2+$ $b-2 a=b$. Now if $n=b+2 a-2$, then $n=b+2$. Hence take $G=G_{5}$ where $\left|V\left(G_{5}\right)\right|=2+2+1+1+(b-2 a)=b+2=n$. If not, take $G=G_{6}$ where $\left|V\left(G_{6}\right)\right|=2+2+1+1+(b-2 a)+(n-b-2 a+2)=n$.
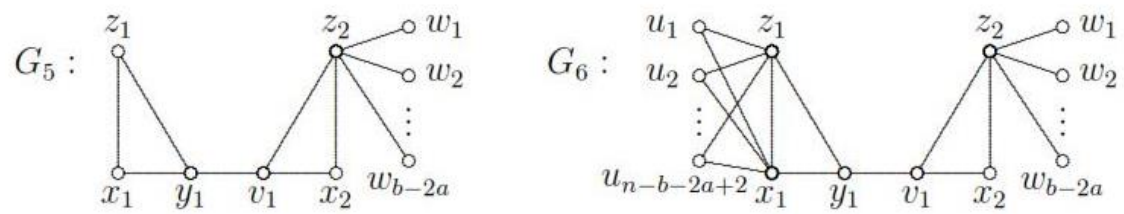

Figure 3: Graphs $G_{5}$ and $G_{6}$

Now suppose $a>2$. Consider the graph $G_{7}$ obtained from $G_{3}$ in Figure 2 by adding the vertices $w_{l}$ and the edges $z_{a} w_{l}$ for $l=1,2, \ldots, b-2 a$. Moreover, let $G_{8}$ be the graph obtained from $G_{7}$ by adding the paths $\left[x_{1}, u_{m}, z_{1}\right]$ for $m=$ $1,2, \ldots, n-b-2 a+2$ (see Figure 4 ). Then the set $\left\{z_{1}, z_{2}, \ldots, z_{a-1}, z_{a}\right\}$ is a $\gamma$-set of $G_{7}$ and $G_{8}$ while a minimum double dominating set of $G_{7}$ and $G_{8}$ is the set $\left\{x_{1}, x_{2}, \ldots, x_{a-1}, x_{a}\right\} \cup\left\{z_{1}, z_{2}, \ldots, z_{a-1}, z_{a}\right\} \cup\left\{w_{1}, w_{2}, \ldots, w_{b-2 a}\right\}$. Hence, $\gamma\left(G_{7}\right)=$ $\gamma\left(G_{8}\right)=a$ and $\gamma_{\times 2}\left(G_{7}\right)=\gamma_{\times 2}\left(G_{8}\right)=a+a+(b-2 a)=b$. Now, if $n=b+2 a-2$, then take $G=G_{7}$. If $n>b+2 a-2$, then take $G=G_{8}$, where $\left|V\left(G_{7}\right)\right|=$ $a+a+(b-2 a)+(a-1)+(a-1)=b+2 a-2=n$ and $\left|V\left(G_{8}\right)\right|=a+a+(n-$ $b-2 a+2)+(b-2 a)+(a-1)+(a-1)=n$.

This proves the assertion.

\section{Double Domination in the Cartesian Product of Graphs}

The Cartesian product $G \square H$ of two graphs $G$ and $H$ is the graph with vertex-set $V(G \square H)=V(G) \times V(H)$ and edge-set $E(G \square H)$ satisfying the following conditions: $(u, v)\left(u^{\prime}, v^{\prime}\right) \in E(G \square H)$ if and only if either $u u^{\prime} \in E(G)$ and $v=v^{\prime}$ or $u=u^{\prime}$ and $v v^{\prime} \in V(H)$.

Theorem 3.1. Let $G$ and $H$ be connected non-trivial graphs. Then $C=$ $\bigcup_{x \in S}\left[\{x\} \times T_{x}\right]$, where $S \subseteq V(G)$ and $T_{x} \subseteq V(H)$ for every $x \in S$, is a double dominating set of $G \square H$ if and only if the following properties hold:

(i) $T_{x}$ is a double dominating set in $H$ for each $x \in S \backslash N(S)$; 

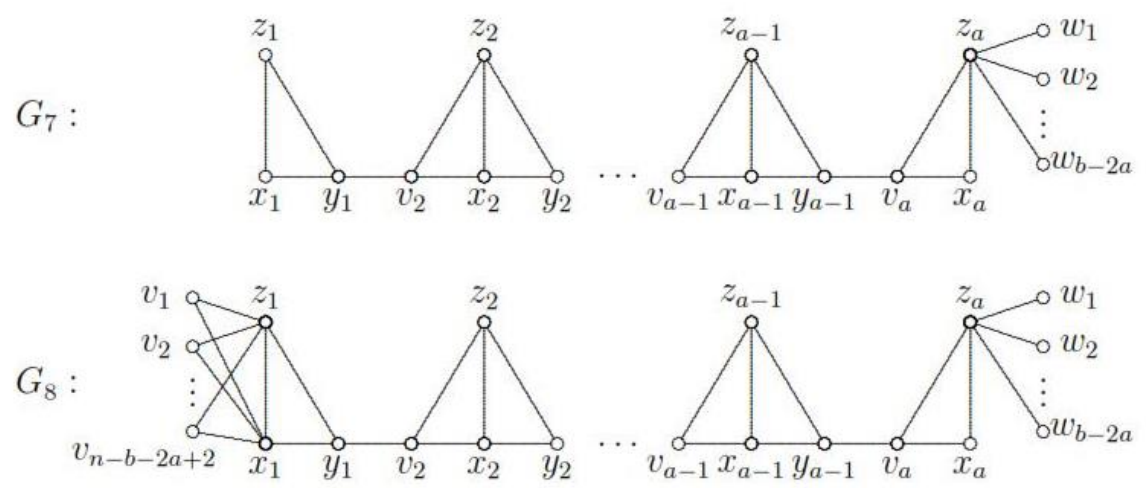

Figure 4: Graphs $G_{7}$ and $G_{8}$

(ii) For each $x \in S \cap N(S)$ and for each $a \in V(H) \backslash N_{H}\left[T_{x}\right]$, there exist $y, z \in$ $N_{G}(x) \cap S$ such that $a \in T_{y} \cap T_{z}$;

(iii) For each $x \in S \cap N(S)$ and for each $a \in N_{H}\left[T_{x}\right]$, one of the following holds:

(a) $a \in T_{x}$ and either $\left|N_{H}[a] \cap T_{x}\right| \geq 2$ or $a \in T_{y}$ for some $y \in N_{G}(x) \cap S$;

(b) $a \notin T_{x}$ and $\left|N_{H}(a) \cap T_{x}\right| \geq 2$ or $a \in T_{y}$ for some $y \in N_{G}(x) \cap S$ and $\left|N_{H}(a) \cap T_{x}\right| \geq 1$ or $a \in T_{y} \cap T_{z}$ for some $y, z \in N_{G}(x) \cap S(y \neq z)$; and

(iv) For each $x \in V(G) \backslash S$ and for each $a \in V(H)$, there exist $y, z \in N_{G}(x) \cap S$, where $y \neq z$, such that $a \in T_{y} \cap T_{z}$.

Proof. Suppose $C$ is a double dominating set of $G \square H$. Let $x \in S \backslash N(S)$ and let $q \in V(H)$. If $q \notin T_{x}$, then $(x, q) \notin C$. Since $C$ is a double dominating set of $G \square H$, there exist distinct vertices $(y, b)$ and $(z, c)$ in $C$ such that $(x, q)(y, b),(x, q)(z, c) \in E(G \square H)$. Since $x \notin N(S)$, it follows that $x=y=z$. Thus $b, c \in N_{H}(q) \cap T_{x}$. If $q \in T_{x}$, then $(x, q) \in C$. Since $C$ is a total dominating set, there exist of $G \square H$, there exists $(y, a) \in C$ such that $(x, q)(y, a) \in E(G \square H)$. Since $x \notin N(S)$, it follows that $x=y$ and $a q \in E(H)$. Hence $a \in T_{x}$ and $a q \in E(H)$. In any case, $\left|N_{H}[q] \cap T_{x}\right| \geq 2$, showing that $T_{x}$ is a double dominating set in $H$.

Next, let $x \in S \cap N(S)$ and let $a \in V(H) \backslash N_{H}\left(T_{x}\right)$. Since $C$ is a double dominating set, there exist distinct vertices $(y, b)$ and $(z, c)$ in $C$ such that $(x, a)(y, b),(x, a)(z, c) \in E(G \square H)$. Since $a \notin N_{H}\left(T_{x}\right), a=b=c$ and $x y, x z \in$ $E(G)$. This implies that $y, z \in N_{G}(x) \cap S$ and $a \in T_{y} \cap T_{z}$.

Now, let $x \in S \cap N(S)$ and let $a \in N_{H}\left[T_{x}\right]$. If $a \in T_{x}$, then $(x, a) \in C$. Since $C$ is a total dominating set, there exists $(y, b) \in C$ such that $(x, a)(y, b) \in E(G \square H)$. Hence $x=y$ and $a b \in E(H)$ or $x y \in E(G)$ and $a=b$. Thus $\left|N_{H}[a] \cap T_{x}\right| \geq 2$ or 
$a \in T_{y}$ for some $y \in N_{G}(x) \cap S$. Suppose $a \notin T_{x}$. Then $a \in N\left(T_{x}\right)$ and $(x, a) \notin C$. Since $C$ is a double dominating set of $G \square H$, there exist $(y, b),(z, c) \in C$ such that $(y, b),(z, c) \in N_{G \square H}((x, a))$. If $x=y=z$, then $b, c \in T_{x} \cap N_{H}(a)$. If either $y$ or $z$ is not $x$, say $x \neq y$, then $x y \in E(G)$ and $a=b \in T_{y}$. If both $y$ and $z$ is not $x(y \neq z)$, then $x y, x z \in E(G)$ and $a=b=c$ with $a \in T_{y} \cap T_{z}$. Thus either $\left|N_{H}(a) \cap T_{x}\right| \geq 2$ or $a \in T_{y}$ for some $y \in N_{G}(x) \cap S$ and $\left|N_{H}(a) \cap T_{x}\right| \geq 1$ or $a \in T_{y} \cap T_{z}$ for some $y, z \in N_{G}(x) \cap S(y \neq z)$

Finally, let $x \in V(G) \backslash S$ and let $a \in V(H)$. Since $C$ is a double dominating set, there exists distinct vertices $(y, b)$ and $(z, c)$ in $C$ such that $(x, a)(y, b),(x, a)(z, c) \in$ $E(G \square H)$. Since $x \notin S, a=b=c$. It follows that $y, z \in N_{G}(x) \cap S$ and $a \in T_{y} \cap T_{z}$.

For the converse, suppose that $S$ is a 2-dominating set in $G$ and that properties (i), (ii), (iii), and (iv) hold. Let $(x, a) \in V(G \square H)$. Consider the following cases:

Case 1. Suppose that $x \notin S$. Then, by (iv) there exist distinct vertices $y$ and $z$ in $N_{G}(x) \cap S$ such that $a \in T_{y} \cap T_{z}$. It follows that $(y, a),(z, a) \in N_{G \square H}((x, a)) \cap C$, where $(y, a) \neq(z, a)$. Thus $\left|N_{G \square H}((x, a)) \cap C\right| \geq 2$.

Case 2. Suppose that $x \in S \backslash N(S)$. By (i), $T_{x}$ is a double dominating set in $H$; hence, there exist two distinct vertices $b, c \in T_{x}$ such that $(x, b),(x, c) \in$ $N_{G \square H}[(x, a)] \cap C$. Thus, $\left|N_{G \square H}[(x, a)] \cap C\right| \geq 2$.

Case 3. Suppose that $x \in S \cap N(S)$ and $a \in V(H) \backslash N\left[T_{x}\right]$. Then $\left|N_{G \square H}((x, a)) \cap C\right| \geq 2$ by (ii).

Case 4. Suppose that $x \in S \cap N(S)$ and $a \in N\left[T_{x}\right]$. Suppose first that $a \notin T_{x}$. Then there exists $b \in T_{x}$ such that $a b \in E(H)$. It follows that $(x, b) \in N_{G \square H}((x, a)) \cap C$. Also, by (iii), either there exists $c \in T_{x} \backslash\{b\}$ or there exists $y \in N_{G}(x) \cap S$ such that $(y, a) \in C$. In either case, we have $\left|N_{G \square H}((x, a)) \cap C\right| \geq 2$. Suppose now that $a \in T_{x}$. Then $(x, a) \in C$. By (iii), it follows that $\left|N_{G \square H}[(x, a)] \cap C\right| \geq 2$.

Case 5. Suppose that $x \in V(G) \backslash S$ and $a \in V(H)$. Then by (iv), $\left|N_{G \square H}((x, a)) \cap C\right| \geq 2$.

Accordingly, $C$ is a double dominating set of $G \square H$.

The following result is immediate.

Corollary 3.2. Let $G$ and $H$ be connected non-trivial graphs of orders $n$ and $m$, respectively. Then,

$$
\gamma_{\times 2}(G \square H) \leq \min \left\{m\left[\gamma_{2}(G)\right], n\left[\gamma_{2}(H)\right]\right\} .
$$

Proof. Let $S$ be a $\gamma_{2}$-set of $G$. Put $T_{x}=V(H)$ for each $x \in S$. Then $C=$ $\bigcup_{x \in S}\left[\{x\} \times T_{x}\right]$ is a double dominating set of $G \square H$ by Theorem 3.1. Hence,

$$
\gamma_{\times 2}(G \square H) \leq|C|=\sum_{x \in S}\left|T_{x}\right|=|S||V(H)|=m \gamma_{2}(G) .
$$

Similarly, $\gamma_{\times 2}(G \square H) \leq n \gamma_{2}(H)$. Thus,

$$
\gamma_{\times 2}(G \square H) \leq \min \left\{m\left[\gamma_{2}(G)\right], n\left[\gamma_{2}(H)\right]\right\} .
$$


Example 3.3. Consider the graphs $P_{5} \square P_{5}$ and $C_{4} \square P_{3}$ in Figure 5. In Figure $5(a)$, the set $\left\{x_{b}, x_{d}, y_{a}, y_{b}, y_{c}, y_{d}, y_{e}, u_{a}, u_{b}, u_{c}, u_{d}, u_{e}, v_{b}, v_{d},\right\}$ is a minimum double dominating set of $P_{5} \square P_{5}$. Thus, $\gamma_{\times 2}\left(P_{5} \square P_{5}\right)=14<15=m\left[\gamma_{2}\left(P_{5}\right)\right]$. This shows that the strict inequality in Corollary 3.2 can be attained. In Figure $5(b)$, the set $\left\{x_{a}, x_{b}, x_{c}, z_{a}, z_{b}, z_{c}\right\}$ is a minimum double dominating set of $C_{4} \square P_{3}$. Hence, $\gamma_{\times 2}\left(C_{4} \square P_{3}\right)=6=m\left[\gamma_{2}\left(C_{4}\right)\right]$. This implies that the bound given in Corollary 3.2 is sharp.

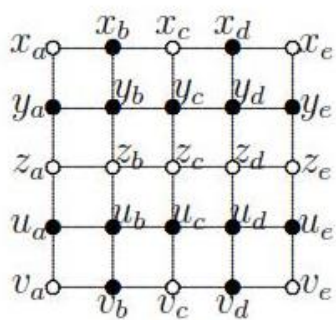

(a) $P_{5} \square P_{5}$

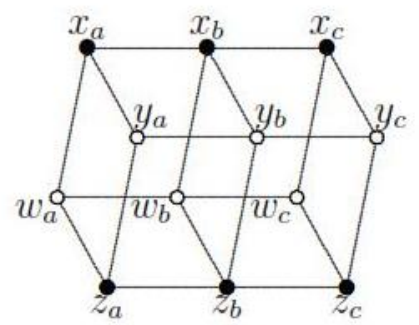

(b) $C_{4} \square P_{3}$

Figure 5: The Cartesian products $P_{5} \square P_{5}$ and $C_{4} \square P_{3}$

\section{Double Domination in the Tensor Product of Graphs}

The Tensor product $G \otimes H$ of two graphs $G$ and $H$ is the graph with vertexset $V(G \otimes H)=V(G) \times V(H)$ and edge-set $E(G \otimes H)$ satisfying the following conditions: $(u, v)\left(u^{\prime}, v^{\prime}\right) \in E(G \otimes H)$ if and only if $u u^{\prime} \in E(G)$ and $v v^{\prime} \in V(H)$.

Theorem 4.1. Let $G$ and $H$ be connected non-trivial graphs. Then $C=$ $\bigcup_{x \in S}\left(\{x\} \times T_{x}\right)$, where $S \subseteq V(G)$ and $T_{x} \subseteq V(H)$ for every $x \in S$, is a double dominating set of $G \otimes H$ if and only if the following properties hold:

(i) There exists $y \in N_{G}(x) \cap S$ with $\left|N_{H}(p) \cap T_{y}\right| \geq 2$ or there exist $y, z \in$ $N_{G}(x) \cap S$, where $y \neq z$, such that $N_{H}(p) \cap T_{y} \neq \phi$ and $N_{H}(p) \cap T_{z} \neq \phi$ whenever $x \in S$ and $p \notin T_{x}$ or $x \notin S$ and $p \in V(H)$; and

(ii) For each $x \in S$ and for each $p \in T_{x}$, there exists $y \in N_{G}(x) \cap S$ such that $\left|N_{H}(p) \cap T_{y}\right| \geq 1$.

Proof. Suppose $C$ is a double dominating set of $G \otimes H$. Suppose $x \in S$ and $p \notin T_{x}$ (or $x \notin S$ and $p \in V(H)$ ). Then there exist at least two vertices $(y, q),(z, t) \in C$ such that $(x, p)(y, q),(x, p)(z, t) \in E(G \otimes H)$. Hence, $x y, x z \in E(G)$ and $p q, p t \in E(H)$. If $y=z$, then $q, t \in N_{H}(p) \cap T_{y}$, where $q \neq t$, and $y \in N_{G}(x) \cap S$. If $y \neq z$, then 
$\left|N_{G}(x) \cap S\right| \geq 2, N_{H}(p) \cap T_{y} \neq \phi$, and $N_{H}(p) \cap T_{z} \neq \phi$. Hence, (i) holds.

Let $x \in S$ and $p \in T_{x}$. Since $C$ is a double dominating set, there exists $(y, q) \in C$ such that $(x, p)(y, q) \in E(G \otimes H)$. It follows that $y \in N_{G}(x) \cap S$ and $\left|N_{H}(p) \cap T_{y}\right| \geq 1$. This shows that (ii) holds.

For the converse, suppose that (i) and (ii) hold. Let $(x, p) \in V(G \otimes H)$ and consider the following cases:

Case 1: $(x, p) \in C$

Then $x \in S$ and $p \in T_{x}$. By (ii), there exists $y \in N_{G}(x) \cap S$ with $\left|N_{H}(p) \cap T_{y}\right| \geq$ 1. Pick any $q \in N_{H}(p) \cap T_{y}$. Then $(y, q) \in C$ and $(x, p)(y, q) \in E(G \otimes H)$. It follows that $\left|N_{G \otimes H}[(x, p)] \cap C\right| \geq 2$.

Case 2: $(x, p) \notin C$

Then either $x \in S$ and $p \notin T_{x}$ or $x \notin S$ and $p \in V(H)$. By $(i)$, suppose there exists $y \in N_{G}(x) \cap S$ such that $\left|N_{H}(p) \cap T_{y}\right| \geq 2$. Pick any $t, q \in N_{H}(p) \cap T_{y}$, where $t \neq q$. Then, $(y, t),(y, q) \in C$ implying that $(x, p)(y, t),(x, p)(y, q) \in E(G \otimes$ $H)$. Hence $\left|N_{G \otimes H}((x, p)) \cap C\right| \geq 2$. Next, suppose that there exist distinct $y, z \in$ $N_{G}(x) \cap S$ with $N_{H}(p) \cap T_{y} \neq \phi$ and $N_{H}(p) \cap T_{z} \neq \phi$. Pick $q \in N_{H}(p) \cap T_{y}$ and $t \in N_{H}(p) \cap T_{z}$. Then, $(y, q)$ and $(z, t)$ are distinct elements of $N_{G \otimes H}((x, p)) \cap C$. Hence $\left|N_{G \otimes H}((x, p)) \cap C\right| \geq 2$.

Accordingly, $C$ is a double dominating set of $G \otimes H$.

Corollary 4.2. Let $G$ and $H$ be connected non-trivial graphs. If $S$ and $D$ are double dominating sets of $G$ and $H$ respectively, then $C=S \times D$ is a double dominating set of $G \otimes H$. In particular,

$$
\gamma_{\times 2}(G \otimes H) \leq \gamma_{\times 2}(G) \gamma_{\times 2}(H) .
$$

Proof. Let $T_{x}=D$ for each $x \in S$. Then $C=\cup_{x \in S}\left[\{x\} \times T_{x}\right]$. By Theorem 4.1, $C$ is a double dominating set of $G \otimes H$. Hence,

$$
\begin{aligned}
\gamma_{\times 2}(G \otimes H) & \leq|C| \\
& =\left|\bigcup_{x \in S}\left[\{x\} \times T_{x}\right]\right| \\
& =|S||D| \\
& =\gamma_{\times 2}(G) \gamma_{\times 2}(H) .
\end{aligned}
$$

This proves the desired result.

Example 4.3. Consider the graphs $P_{6} \otimes P_{6}$ and $P_{5} \otimes P_{5}$ in Figure 6. In Figure $6(a)$, it can be seen that $\gamma_{\times 2}\left(P_{6} \otimes P_{6}\right)=20<25=\gamma_{\times 2}\left(P_{6}\right) \gamma_{\times 2}\left(P_{6}\right)$. In Figure $6(b), \gamma_{\times 2}\left(P_{5} \otimes P_{5}\right)=16=\gamma_{\times 2}\left(P_{5}\right) \gamma_{\times 2}\left(P_{5}\right)$. These graphs show that both the strict inequality and equality in Corollary 4.2 can be attained. 


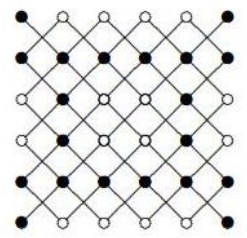

(a) $P_{6} \otimes P_{6}$

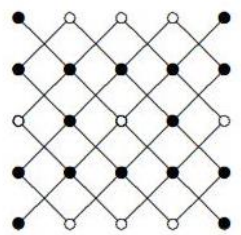

(b) $P_{5} \otimes P_{5}$

Figure 6: The tensor products of $P_{6} \otimes P_{6}$ and $P_{5} \otimes P_{5}$

\section{References}

[1] M. Atapour, A. Khodkar and S. M. Sheikholeslami, Characterization of double domination subdivision number of trees, Discrete Applied Mathematics, 155(2007), 17001707.

[2] M. Blidia, M. Chellali and T. W. Haynes, Characterization of trees with equal paired and double domination numbers, Discrete Mathematics, 306(2006), 1840-1845.

[3] A. Cuivillas and S. Canoy, Double domination in graphs under some binary operations, Applied Mathematical Sciences, 8(2014), 2015-2024.

[4] F. Harary and T. W. Haynes, Double domination in graphs, Arts Combin, 55(2000), 201-213.

[5] F. Harary and T. W. Haynes, Norhdhaus-Gaddum inequalities for domination in graphs, Discrete Mathematics, 155(1996), 99-105.

[6] S. Khelifi, M. Blidia, M. Chellali and F. Maffrey, Double domination edge removal critical graphs, Australasian Journal of Combinatorics, 48(2010), 285-299. 